\title{
(1)
}

UNIVERSIDAD PERUANA DE CIENCIAS APLICADAS

FACULTAD DE CIENCIAS DE LA SALUD

ESCUELA DE MEDICINA HUMANA

\section{"Razón Mortalidad-Incidencia como indicador de supervivencia de cáncer a cinco años en Lima Metropolitana"}

\author{
TESIS \\ Para Optar por el título de MEDICO CIRUJANO \\ Autores: \\ Alejandra Cordero Morales \\ Maria Jose Savitzky Franco \\ Karoline Andrea Stenning Persivale
}

Asesores:

Eduardo Payet Meza

Eddy Segura Paucar

Lima 2017 
"Si caminas solo iras más rápido, si caminas acompañado llegarás más lejos" - Proverbio Chino. Para todos los que nos acompañaron en este largo camino. 


\section{AGRADECIMIENTOS}

Gracias a Dios por guiarnos todos estos años y a nuestras familias por su apoyo incondicional.

Gracias a nuestros amigos que hicieron las clases más divertidas y a aquellos que supieron entender el "no puedo, tengo guardia"

Gracias a nuestros asesores y al Instituto Nacional de Enfermedad Neoplásicas por hacer esta tesis posible.

Gracias a la facultad de Ciencias de la Salud de la UPC la formación brindada y su compromiso a nosotras 


\section{RESUMEN}

Introducción: El complemento de la Razón Mortalidad-Incidencia [1 - RMI], ha sido sugerido como un proxy válido para hallar la supervivencia a cinco años de cáncer. Su validez aún no ha sido reportada en el Perú. El presente estudio busca establecer si esta razón guarda una correlación directa con la supervivencia de los pacientes con cáncer a cinco años en Lima Metropolitana. Materiales y Métodos: Se utilizó el Registro de Cáncer de Lima Metropolitana (RCLM) del 2004-2005 de donde se obtuvo la incidencia y mortalidad por tipo de neoplasia y la supervivencia observada. A partir de estas, se buscó correlacionar [1 - RMI] con la supervivencia observada a cinco años de diez tipos de neoplasias seleccionadas (Mama, estómago, próstata, tiroides, pulmón, colon, linfoma no Hodgkin, cuello uterino, hígado y leucemia) mediante el modelo de regresión lineal. Resultados: Considerando los diez tipos de cáncer estudiados, se realizó una regresión lineal en donde se encontró correlación entre la supervivencia observada a cinco años y [1-RMI]. De las diez neoplasias, cinco tuvieron una correlación muy alta, siendo estas: Mama, próstata, hígado, tiroides y leucemia. Conclusión: El complemento de la Razón Mortalidad Incidencia [1-RMI] es una buena herramienta para la aproximación de la supervivencia observada a cinco años para los diez tipos de neoplasias estudiadas. Demostrándose la validez del modelo como predictor de supervivencia a cinco años en pacientes con cáncer, para Lima Metropolitana.

Palabras clave: Perú, Mortalidad, Incidencia, Cáncer, Supervivencia, Registros de Cáncer. 


\section{ABSTRACT}

"Mortality to Incidence Ratio as a proxy for five-year cancer survival in Metropolitan Lima"

Introduction: Mortality-Incidence Ratio complement [1 - (M / I)] has been suggested as a valid proxy to find the five-year cancer survival. Its validity has not been assessed in Peru, so this study seeks to establish whether this ratio has a direct correlation with five-year cancer survival in Metropolitan Lima. Methods: The Metropolitan Lima Cancer Registry of 2004-2005 was used to obtain the incidence and mortality rates by type of neoplasia and survival rates. From these, we looked to correlate [1 - (M / I)] with five-year observed survival rates of ten types of selected cancers (Breast, stomach, prostate, thyroid, lung, colon, non-Hodgkin lymphoma, cervical, liver and leukemia), using a linear regression model. Results: Considering the types of cancer studied, the regression line approximates the perfect line with indicating a good correlation between the studied parameters. The types of neoplasm that obtained a better correlation between [1- (M / I)] and observed survival were: Breast, prostate, liver, thyroid and leukemia. Conclusion: The Mortality- Incidence Ratio complement [1- (M / I)] is a good tool for approximating the five-year survival rate for the ten types of cancer studied. This study demonstrates the validity of the method, showing that the model can be applied in Metropolitan Lima and for the types of malignancies studied.

Key words: Peru, Mortality, Incidence, Cancer, Survival, Cancer registries. 


\section{TABLA DE CONTENIDO}

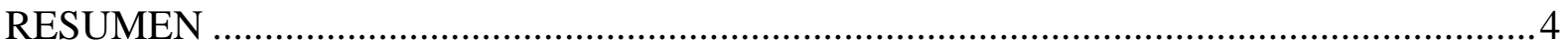

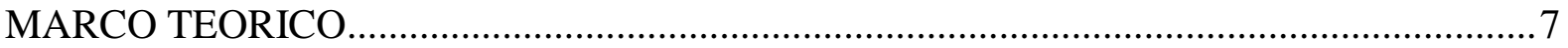

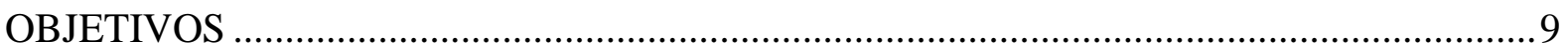

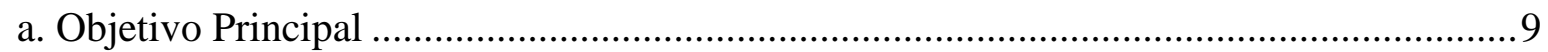

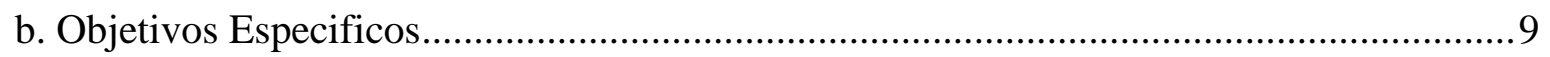

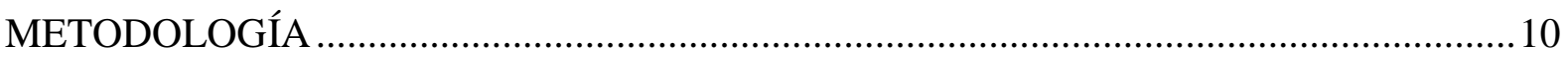

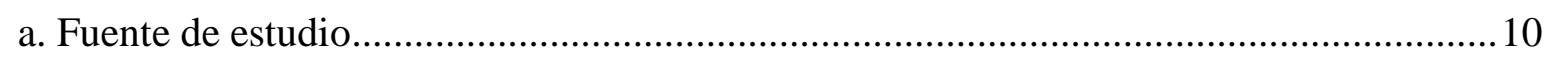

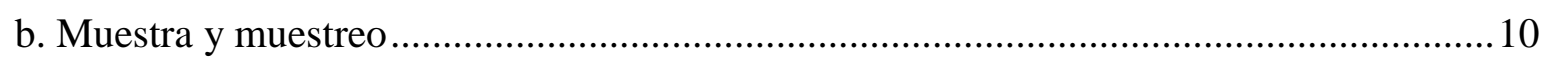

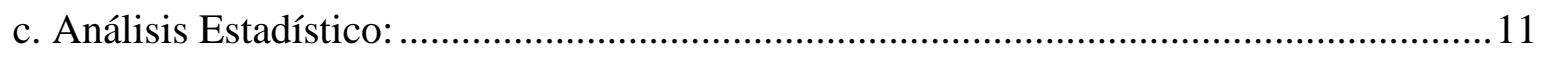

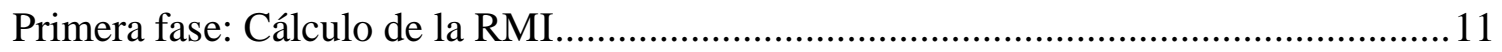

Segunda fase: Análisis de supervivencia observada ........................................................ 12

Tercera fase: Análisis de correlación entre [1-RMI] y la supervivencia observada a 5 años

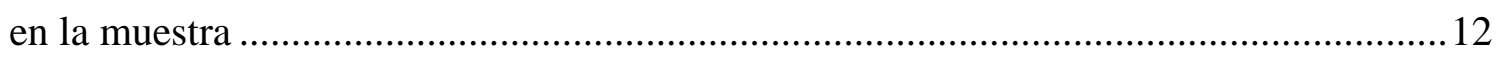

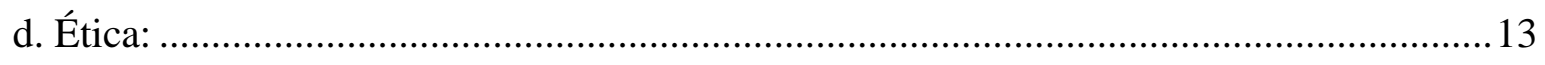

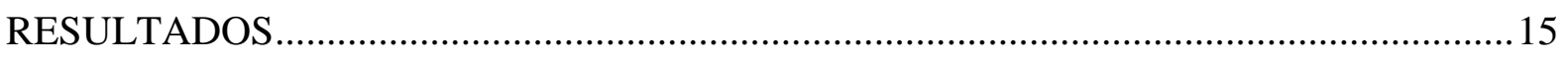

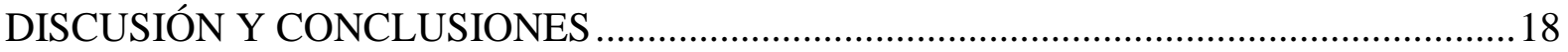

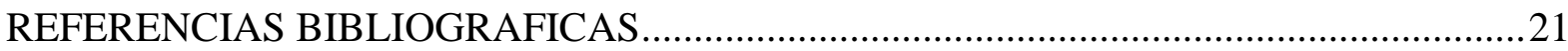

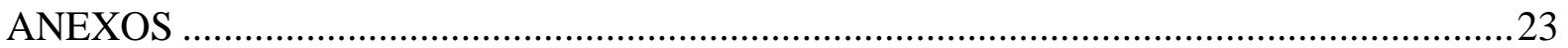




\section{MARCO TEÓRICO}

El cáncer es una de las enfermedades más devastadoras y figura entre las principales causas de morbilidad y mortalidad en todo el mundo. Según la Organización Mundial de la Salud (OMS), 8.2 millones de personas a nivel mundial fallecieron a causa de cáncer en el año 2012. ${ }^{1}$ En el Perú, esta enfermedad es uno de los principales problemas de salud pública y ocupa la segunda causa de muerte en ambos sexos, después de las enfermedades cardiovasculares. ${ }^{2}$ Por tratarse de una enfermedad con posibilidades de ser prevenida y potencialmente curable, es importante disponer de indicadores como: incidencia, prevalencia, mortalidad y supervivencia, que permitan hacer un seguimiento de la enfermedad, evaluar su estado actual y de esta manera asignar recursos para las estrategias de control de la misma.

Investigar la supervivencia es importante para evaluar el comportamiento del cáncer y la respuesta de los sistemas de salud frente a este. ${ }^{3,4}$ Por otro lado, la supervivencia es útil para formular estrategias de lucha contra el cáncer y evaluar la eficacia y rentabilidad de estas. ${ }^{5} \mathrm{El}$ conocimiento de supervivencia es esencial para la planificación y la gestión de la enfermedad, así como para ayudar a monitorizar y controlar los factores de pronóstico en la población. ${ }^{6,7}$ Para poder estimar la supervivencia a nivel nacional se necesita una base que obtenga información completa y confiable de una región específica, tal como lo son los registros de cáncer de base poblacional (RCBP). En el Perú, solo se cuenta con un registro que cumple dichos criterios, el Registro de Cáncer de Lima Metropolitana (RCLM), el cual recolecta información de todos los casos de cáncer en Lima ${ }^{8}$. Este registra la incidencia y mortalidad de cáncer en la población en estudio durante un determinado tiempo, y da a conocer diferentes aspectos de la enfermedad en la población. Para estos registros, es difícil realizar estudios de supervivencia, debido a la dificultad en el seguimiento activo de los pacientes con cáncer, el financiamiento y la logística necesaria para llevar a cabo un estudio de este tipo en un país en desarrollo como el Perú.

En la actualidad, existe un indicador que puede ser utilizado para estudiar la supervivencia, este es el complemento de la Razón Mortalidad-Incidencia (RMI). La RMI es una división del 
número de muertes sobre el número de nuevos casos de un tipo específico de cáncer, registrado en un mismo periodo de tiempo y población específica. ${ }^{4,9,10}$ En el año 2010, en Holanda, se estudió la validez de la RMI como un indicador de la supervivencia para cada tipo de cáncer en los países estudiados. En este estudio se analizaron siete registros de cáncer de distintos países y se utilizó data de treinta y dos tipos de cáncer, concluyendo que la RMI puede ser utilizada para estimar la supervivencia a 5 años en los tipos de neoplasias y población estudiada. $^{3}$

El presente estudio busca evaluar la validez de la Razón Mortalidad-Incidencia, como predictor de la supervivencia de los pacientes con cáncer en Lima Metropolitana. 


\section{OBJETIVOS}

\section{a. Objetivo Principal}

Evaluar si la Razón Mortalidad-Incidencia es útil como predictor de la supervivencia de los pacientes con cáncer en Lima Metropolitana a cinco años.

\section{b. Objetivos Específicos}

Establecer la Razón Mortalidad-Incidencia de los pacientes con neoplasias malignas en Lima Metropolitana utilizando la base de datos del Registro de Cáncer de Lima Metropolitana (RCLM).

Determinar la supervivencia a cinco años de las neoplasias malignas determinadas para el presente estudio 


\section{METODOLOGÍA}

\section{a. Fuente de estudio}

Para este estudio se utilizó la data del RCLM, correspondiente a los casos de los años 20042005 elaborado por el Instituto Nacional de Enfermedades Neoplásicas (INEN) ${ }^{8}$. Esta base poblacional incluye a los pacientes que residen en Lima Metropolitana (Lima y Callao) diagnosticados con cáncer durante el periodo 2004-2005, así como también a los pacientes fallecidos con cáncer en el mismo periodo. De este, se obtuvieron los datos de casos nuevos, la mortalidad y la RMI. Este RCBP ha pasado por un sistema de control de calidad de los datos recolectados y procesados, por lo que fue certificado a nivel internacional.

Respecto a los datos de supervivencia observada, se generó una nueva base de datos para verificar el estado vital a cinco años de cada caso de la muestra en estudio a partir del RCLM. Estos datos se obtuvieron utilizando el acceso al Registro Nacional de Identificación y Estado Civil (RENIEC) mediante el personal autorizado del departamento de Epidemiología y Estadística de Cáncer del INEN de acuerdo a su convenio vigente con el INEN. Para mantener la confidencialidad, se utilizó un sistema de código para cada caso seleccionado.

\section{b. Muestra y muestreo}

Se incluyeron diez neoplasias malignas (Mama, estómago, próstata, cérvix, pulmón, linfoma no Hodgkin, colon, leucemia, tiroides e hígado), estas representan a las de aparición más frecuente, mortalidad más alta y la más baja. ${ }^{8}$ Posteriormente, se retiraron los casos cuyos certificados de defunción fue método de diagnóstico ya que ello perjudica el cálculo la supervivencia. ${ }^{11}$ 
Para aquellas neoplasias que superaron los mil casos nuevos, se calculó un tamaño de muestra representativa utilizando un muestreo aleatorio simple sin reemplazo. Así mismo, se incrementó el tamaño muestral en un $10 \%$ ante la posibilidad de no encontrar la totalidad de los casos escogidos. (Tabla 1)

\section{Tabla 1. Tabla de diez neoplasias utilizadas con su tamaño de muestra}

\begin{tabular}{cccc} 
Neoplasia & $\begin{array}{c}\text { Casos Nuevos } \\
\text { modificados }^{*}\end{array}$ & $\begin{array}{c}\text { Tamaño De } \\
\text { Muestra }\end{array}$ & $\begin{array}{c}\text { Tamaño De } \\
\text { Muestra Final }\end{array}$ \\
\hline Mama & 2749 & 548 & 603 \\
Estómago & 2235 & 517 & 569 \\
Próstata & 2342 & 554 & 610 \\
Cérvix & 1602 & 517 & 569 \\
Pulmón & 1255 & 353 & 389 \\
Linfoma No Hodgkin & 1423 & 506 & 557 \\
Colon & 1047 & 449 & 494 \\
Leucemia & 746 & - & - \\
Tiroides & 743 & - & - \\
Hígado & 301 & - & -
\end{tabular}

*Casos modificado: Nuevos casos - aquellos cuyo diagnóstico fue por acta de defunción.

**Muestra final $=$ Tamaño de muestra $+10 \%$

-No Aplica (No se calculó tamaño de muestra, se utilizó todos los casos)

Por último, la selección de los casos se realizó de manera sistemática. (Ver sección Muestra y Muestreo en ANEXOS).

\section{c. Análisis Estadístico:}

\section{Primera fase: Cálculo de la RMI}

Con el RCLM se utilizó el número de muertes obtenido y el número de casos nuevos registrados por cada tipo de cáncer. De esta manera se establece la RMI. 


$$
\mathrm{RMI}=\frac{\text { Número de muertes }}{\text { Número de nuevos casos }}
$$

Con este dato, se calculó el complemento [1 - RMI] es un número con valores entre 0 y 100\%, donde una aproximación al $0 \%$ representa una pobre supervivencia y una aproximación a 100 $\%$ representa una excelente supervivencia. Los valores obtenidos se pueden observar en la Tabla 2.

\section{Segunda fase: Análisis de supervivencia observada}

Para obtener la supervivencia, se utilizó el método de Kaplan-Meier. Se consideraron como vivos aquellos casos cuyo último control se realizó después de los 5 años del diagnóstico y como fallecidos a aquellos casos cuya fecha de defunción según la RENIEC se encontraba dentro de los 5 años de realizado el diagnóstico. Con la ayuda del programa IBM SPSS Statistics 22 se obtuvo la probabilidad de supervivencia para cada neoplasia en un período de cinco años. Este análisis muestra las curvas de supervivencia para cada una de las neoplasias, donde el eje "Y" representa la supervivencia observada en términos de probabilidad y el eje "X" el tiempo de supervivencia en años. Se dividió el análisis en dos gráficos: Gráfico 1 a donde muestra la supervivencia observada de las cinco neoplasias más frecuentes y Gráfico 1b con las cinco neoplasias restantes para su mejor visualización y análisis.

\section{Tercera fase: Análisis de correlación entre [1-RMI] y la supervivencia observada a 5 años en la muestra}

Para medir la correlación entre estas dos variables, usamos el coeficiente de Pearson ${ }^{\circledR}$. Para ello consideraremos la variable [1-RMI] (X) y la variable Supervivencia observada (Y). El coeficiente r obtenido es útil para valorar la correlación entre dos variables, y existen valores estándar para dicho coeficiente. En donde un valor r entre 0,7 y 1 indica una buena correlación, y un $r$ entre 0,2 y 0,4 una baja correlación. 
Posteriormente se construyó un modelo de regresión lineal para explicar el comportamiento de la supervivencia observada en función a [1-RMI] utilizando los datos obtenidos con la siguiente ecuación: $Y=a+b$ X. (Observar Gráfico 2).

\section{d. Ética:}

El estudio se presentó y fue aceptado por el comité de ética de la Universidad Peruana de Ciencias Aplicadas (UPC) al igual que al comité de ética del Instituto Nacional de Enfermedades Neoplásicas (INEN). Este no tiene afectación alguna sobre los pacientes con diagnóstico de cáncer ya que no se trabajó con ellos sino directamente con el RCLM y sus bases de datos.

Para el acceso de los datos de la supervivencia observada se utilizó el convenio y autorización de la RENIEC con el INEN. En la búsqueda de los datos para mantener la confidencialidad, se utilizó un sistema de código para cada caso seleccionado y se accedió a estos desde el INEN. 



\section{RESULTADOS}

En la Tabla 2, se observan los valores obtenidos para la RMI y su complemento [1-RMI]; así como también los valores de la supervivencia observada a 5 años. Entre estos valores se evidencia que los cánceres con mayor porcentaje de supervivencia estimada [1-RMI] a cinco años son: Tiroides (86,7\%), Mama (68\%), Próstata (63,8\%) y Cérvix (58,5\%). Así mismo, las neoplasias con menor porcentaje de supervivencia estimada son: Pulmón (19,4\%), Hígado $(12,2 \%)$, Leucemia $(33,2 \%)$ y Estómago $(31,1 \%)$. Estos resultados fueron obtenidos a partir del complemento de la RMI calculada con los datos del RCLM.

Tabla 2. Tabla con resultados del Complemento de la Razón Mortalidad-Incidencia [1-RMI] y la supervivencia observada

\begin{tabular}{|c|c|c|c|c|c|}
\hline Neoplasia & Defunciones & $\begin{array}{l}\text { Casos } \\
\text { Nuevos }\end{array}$ & RMI & $\begin{array}{l}\text { Supervivencia } \\
(1-\mathrm{RMI}) \quad(\mathrm{X})\end{array}$ & $\begin{array}{c}\text { Supervivencia } \\
\text { observada A } 5 \text { años (Y) }\end{array}$ \\
\hline Mama & 925 & 2886 & $32,0 \%$ & $68,0 \%$ & $69,6 \%$ \\
\hline Estómago & 1950 & 2831 & $68,9 \%$ & $31,1 \%$ & $21,8 \%$ \\
\hline Próstata & 993 & 2744 & $36,2 \%$ & $63,8 \%$ & $64,3 \%$ \\
\hline Cérvix & 687 & 1656 & $41,5 \%$ & $58,5 \%$ & $50,1 \%$ \\
\hline $\begin{array}{c}\text { Tráquea, Bronquios Y } \\
\text { Pulmón }\end{array}$ & 1366 & 1695 & $80,6 \%$ & $19,4 \%$ & $8,2 \%$ \\
\hline Linfoma No Hodgkin & 776 & 1533 & $50,6 \%$ & $49,4 \%$ & $42,0 \%$ \\
\hline Colon & 638 & 1248 & $51,1 \%$ & $48,9 \%$ & $41,8 \%$ \\
\hline Leucemia & 625 & 935 & $66,8 \%$ & $33,2 \%$ & $31,0 \%$ \\
\hline Tiroides & 101 & 760 & $13,3 \%$ & $86,7 \%$ & $86,7 \%$ \\
\hline Hígado & 576 & 656 & $87,8 \%$ & $12,2 \%$ & $12,6 \%$ \\
\hline
\end{tabular}

Así mismo, en la Tabla 2 se observa los resultados de la supervivencia observada a partir de la base de datos generada en base al acceso a la RENIEC y que se observan en las gráficas $1^{\text {a }}$ y $1 \mathrm{~b}$ donde cada curva equivale a un tipo de cáncer y la supervivencia observada según el tiempo (eje $\mathrm{X})$. Entre estos valores se evidencia que los cánceres con mayor porcentaje de supervivencia observada a cinco años son: Tiroides (86,7\%), Mama (69,6\%), Próstata $(64,3 \%)$ 
y Cérvix (50,1\%). Así mismo, las neoplasias con menor porcentaje de supervivencia observada son: Pulmón (8,2\%), Hígado (12,6\%), Estómago (21,8\%) y Leucemia (31,0\%); siendo estos similares a la supervivencia obtenida [1-RMI].

Gráfico 1a. Curvas de supervivencia observada para las cinco neoplasias más frecuentes

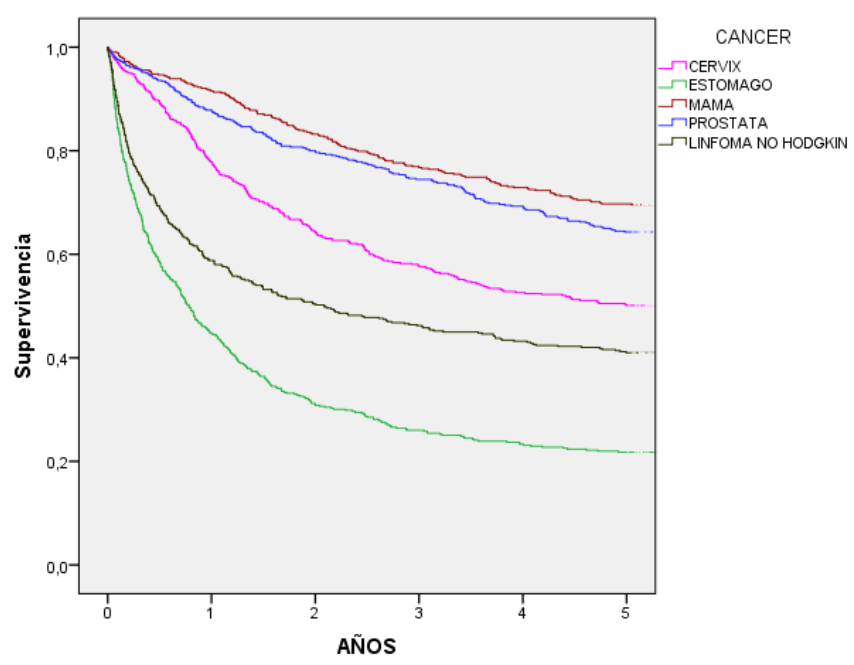

Gráfico 1b. Curvas de supervivencia observada para las cinco neoplasias restantes

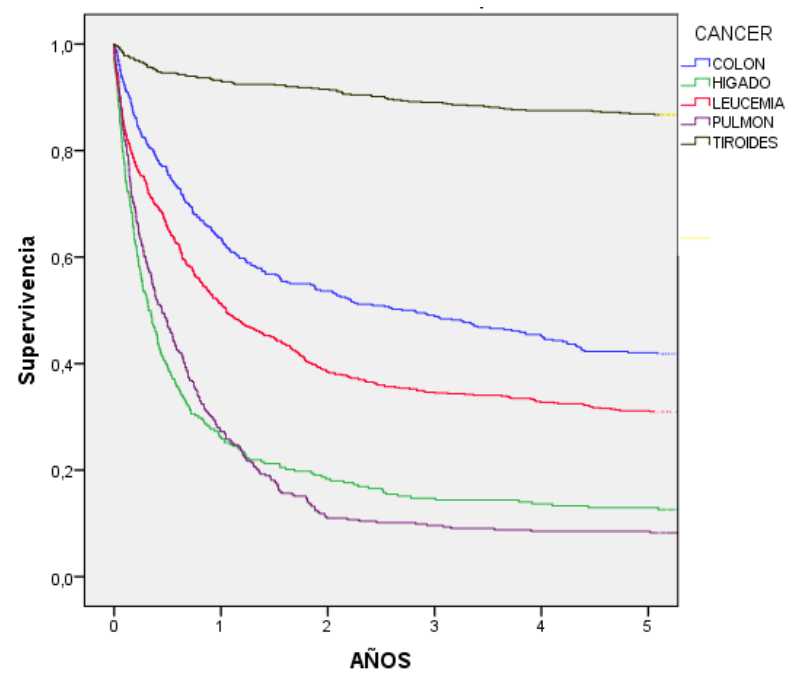




\section{Gráfico 2. Modelo de regresión lineal de la supervivencia observada}

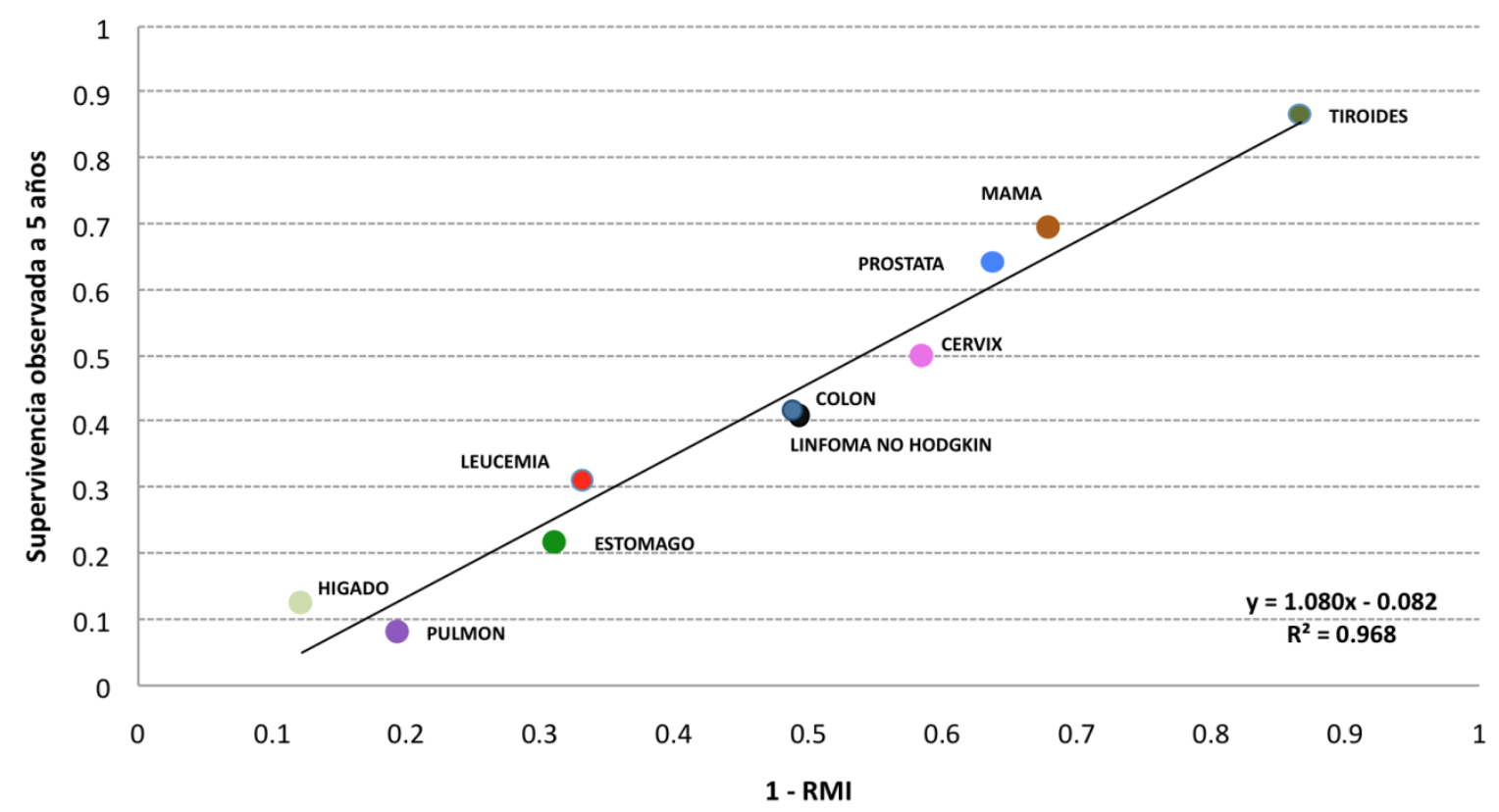

Para medir la correlación entre estas dos variables, [1-RMI] (X) y la variable Supervivencia observada (Y), se utilizó el coeficiente de Pearson ${ }^{\circledR}$ el cual mostró una buena correlación de las variables estudiadas con un valor de $\mathrm{r}=0,9839$. Con los datos previos, se obtuvo el siguiente modelo de regresión lineal $\mathrm{Y}=1,0807 \mathrm{X}$ - 0,082 (Gráfico 2). Con esta información, se halló que el coeficiente de determinación, $\mathrm{R}^{2}=96,82 \%$. Lo cual indica que el $96,82 \%$ de las variaciones en la supervivencia observada $(\mathrm{Y})$ son explicadas por las variaciones de [1-RMI] $(\mathrm{X})$.

Al observar el Gráfico 2, se pueden apreciar los distintos tipos de neoplasias; donde los que obtuvieron una mejor aproximación de la supervivencia observada en función de [1-RMI] son: Mama (1,7\%), Próstata (0,5\%), Hígado $(0,4 \%)$, Tiroides $(0,0 \%)$ y Leucemia $(2,2 \%)$. Donde estos valores presentados, son los puntos porcentuales de diferencia entre la supervivencia observada a cinco años y el complemento [1-RMI]. El resto de neoplasias tampoco mostraron una diferencia significativa, pero las 5 neoplasias mencionadas presentan las diferencias más bajas del grupo estudiado. 


\section{DISCUSIÓN Y CONCLUSIONES}

La RMI ha sido utilizada en diversos estudios en el mundo como indicador de supervivencia a cinco años para diferentes tipos de cáncer. Parkin et al afirman que, en un estado constante de recolección de datos estricto sobre mortalidad e incidencia de las neoplasias, dicha razón es un buen indicador de supervivencia a 5 años. ${ }^{10}$ Sin embargo, según nuestro conocimiento, nunca se ha realizado un estudio que utilice esta razón como instrumento de evaluación de supervivencia en nuestro país. Debido a que la incidencia y mortalidad de cáncer varían según el estilo de vida, genética, medio ambiente, acceso a un sistema de salud, acceso a tratamiento, entre otros, es que surgió la necesidad de evaluar si este indicador es válido para la población de Lima Metropolitana.

En salud pública, la RMI es una herramienta necesaria para entender patrones y riesgo de cáncer en una población y establecer el comportamiento y carga de cada neoplasia. Esta ayuda a predecir la carga futura, tendencias, y por lo tanto sacar proyecciones de cada neoplasia y mantener una vigilancia sobre estas, algo que resulta vital en la oncología. ${ }^{4,13}$ Esta razón servirá para evaluar de manera retrospectiva que poblaciones presentaron menor o mayor supervivencia a 5 años, y así relacionar esta información con los métodos de prevención, diagnóstico y tratamiento en ese mismo periodo de tiempo. De esta manera se podrá conocer qué acciones se llevaron a cabo en ese momento y así enfocarse en la toma de decisiones para lograr una mayor supervivencia a futuro. ${ }^{12,14}$

Al colocar las variables [1-RMI] y supervivencia observada en el modelo de regresión lineal se puede observar que los diez tipos de cáncer estudiados en la línea de regresión permanecen cercanos a línea perfecta y =x, lo que podría indicar una buena correlación. Posteriormente se obtuvo el coeficiente de determinación: $\mathrm{R}^{2}=0,9682$, lo cual indica que el $96,82 \%$ de las variaciones en la supervivencia observada $(\mathrm{Y})$ son explicadas por las variaciones de [1-RMI] 
(X). De esta forma, se demuestra que la RMI es un proxy válido para la supervivencia a 5 años para los tipos de neoplasias estudiadas en la población de Lima Metropolitana.

Si bien los resultados obtenidos corroboran el uso de la RMI para evaluar la supervivencia de cáncer a cinco años, también es importante reconocer las limitaciones de este estudio. La RMI sólo puede ser utilizada de manera válida con un RCBP y elaborar este registro puede resultar igual de complicado que el seguimiento activo de casos para realizar estimaciones reales de supervivencia. En el Perú solo se cuenta con un RCBP que es el RCLM, el cual recolecta data de todos los hospitales públicos y privados de la región Lima Metropolitana. ${ }^{8}$ La labor que realiza el INEN para llevar a cabo el RCLM es compleja y necesita de personal, logística y presupuesto; es por estas dificultades que no se ha realizado este trabajo en otras ciudades del país ni se ha analizado data de otros años. Esto resulta en una limitación ya que el cálculo de supervivencia basado en la RMI dependerá de la posibilidad de que el INEN u otra institución continúe con la recolección de datos y elaboración de RCBP a lo largo de los años. Sin embargo, esta realidad no es igual para todos los países, un claro ejemplo es Brasil. En dicho país cuentan con registros actualizados de información, los cuales contienen data importante que permite realizar un seguimiento activo de los pacientes a lo largo del tiempo. ${ }^{7}$

Otra limitación se presentó al trabajar con data del RENIEC para corroborar la supervivencia de los casos seleccionados ya que un porcentaje de estos no cuentan con la información actualizada, pudiendo generar un sub-registro respecto a los casos estudiados de la muestra. ${ }^{11}$ Es por este motivo que durante el desarrollo del estudio se incrementó el tamaño muestral. Al mismo tiempo, otra limitación fue que no se calculó la RMI para todas las neoplasias y solo se hizo para diez tipos de neoplasias por razones de tiempo. Si bien diez neoplasias es un número pequeño para el modelo de regresión lineal se decidió comenzar con estas diez neoplasias representativas para luego complementar con estudios más extensos, dando un punto de partida. A pesar de ello, se debe tener presente que se estudió neoplasias que más representaban a la población (las de mortalidad más alta y más baja, las de mayor incidencia y neoplasias presentes en cada sexo). Al finalizar el estudio se puede concluir que esta razón puede ser utilizado para estas pero no generalizar para todas las neoplasias en Lima Metropolitana. Si 
bien esta es una limitación, al probar que la RMI funciona para estas diez neoplasias damos un punto de partida para nuevos estudios y utilidades de la RMI. ${ }^{13}$

Finalmente, con lo mencionado previamente se puede concluir que las diferencias entre la supervivencia estimada por la RMI y la supervivencia observada no son significativas, por lo que la RMI parece ser un predictor adecuado de la supervivencia a cinco años en los tipos de cáncer estudiados en Lima-Metropolitana, confirmando así su validez únicamente para esta población. Por ello, este estudio otorga una nueva herramienta para estudio de esta población a futuro en las áreas de oncología, epidemiología, salud pública, gestión de salud, entre otras. ${ }^{15}$ 


\section{REFERENCIAS BIBLIOGRAFICAS}

1. International agency for Research on Cancer. World Cancer Report 2014. Ginebra, Suiza: World Health Organization , 2014.

2. Instituto Nacional de Enfermedades Neoplásicas. Guías para la promoción de la salud orientada a la prevención y control del Cáncer. Lima; Peru: Instituto Nacional de Enfermedades Neoplásicas. Diciembre 2006.

3. Asadzadeh Vostakolaei F, Karim-kos HE, Janssen-heijnen ML, Visser O, Verbeek AL, Kiemeney LA. The validity of the mortality to incidence ratio as a proxy for sitespecific cancer survival. Eur J Public Health. 2011;21(5):573-7.

4. Sunkara V, Hébert JR. The colorectal cancer mortality-to-incidence ratio as an indicator of global cancer screening and care. Cancer. 2015;121(10):1563-9.

5. Allemani C, Weir HK, Carreira H, et al. Global surveillance of cancer survival 19952009: analysis of individual data for $25,676,887$ patients from 279 population-based registries in 67 countries (CONCORD-2). Lancet. 2015;385(9972):977-1010.

6. Pruegsanusak K, Peeravut S, Leelamanit V, et al. Survival and prognostic factors of different sites of head and neck cancer: an analysis from Thailand. Asian Pac J Cancer Prev. 2012;13(3):885-90.

7. Schneider I, Flores ME, Nickel DA, Martins LG, Traebert J. Survival rates of patients with cancer of the lip, mouth and pharynx: a cohort study of 10 years. Rev Bras Epidemiol. 2014;17(3):680-91.

8. Instituto Nacional de Enfermedades Neoplásicas. Registro de Cáncer de Lima Metropolitana: Estudio de Incidencia y Mortalidad 2004-2005. Lima, Peru: INEN, 2014. 
9. Parkin DM, Hakulinen T. Cancer registration: principles and methods. Analysis of survival. IARC Sci Publ. 1991;(95):159-76.

10. Parkin DM, Bray F. Evaluation of data quality in the cancer registry: principles and methods Part II. Completeness. Eur J Cancer. 2009;45(5):756-64.

11. Robinson D, Sankila R, Hakulinen T, Møller H. Interpreting international comparisons of cancer survival: the effects of incomplete registration and the presence of death certificate only cases on survival estimates. Eur J Cancer. 2007;43(5):909-13.

12. Maruvka Y, Tang M, Michor F. On the Validity of Using Increases in 5-Year Survival Rates to Measure Success in the Fight against Cancer. PLoS ONE. 2014;9(7):e 83100.

13. Hébert J, Daguise V, Hurley D. Mapping cancer mortality-to-incidence ratios to illustrate racial and sex disparities in a high-risk population. Cancer. 2009;115(11):2539-2552.

14. Wagner SE, Hurley DM, Hébert JR, Mcnamara C, Bayakly AR, Vena JE. Cancer mortality-to-incidence ratios in Georgia: describing racial cancer disparities and potential geographic determinants. Cancer. 2012;118(16):4032-45.

15. Batouli A, Jahanshahi P, Gross CP, Makarov DV, Yu JB. The global cancer divide: Relationships between national healthcare resources and cancer outcomes in highincome vs. middle- and low-income countries. J Epidemiol Glob Health. 2014 Jun;4(2):115-24. 


\section{ANEXOS \\ Muestra y muestreo}

Procedimiento para el cálculo de tamaño de muestra para el estudio de supervivencia:

Se incluyeron 10 neoplasias malignas (Mama, estómago, próstata, cérvix, pulmón, linfoma no Hodgkin, colon, leucemia, tiroides e hígado), estas representan a las de aparición más frecuente, así como también aquellas que presentan la mortalidad más alta y la más baja. ${ }^{8}$

El paso inicial fue retirar los casos cuyos diagnósticos de cáncer fueron obtenidos por medio de los certificados de defunción ya que ello perjudica el cálculo del tiempo de supervivencia. ${ }^{11}$ A esto se le denominó: casos nuevos modificados. Por otro lado, considerando que el número de casos nuevos para cada neoplasia fue muy variable se determinó que para aquellas neoplasias que superaron los mil casos registrados, se debía realizar el cálculo del tamaño de muestra representativa para el estudio. Aquellas neoplasias en las cuales los casos registrados fueron menor de mil (Hígado, Leucemia y Tiroides), se procedió a utilizar a toda la población para el estudio de supervivencia.

El tamaño de muestra requerido en cada una de estas neoplasias se calculó utilizando un muestreo aleatorio simple sin reemplazo; donde el tamaño de muestra requerido está determinado por la siguiente fórmula:

$\mathrm{n}=\frac{N * P * Q}{(N-1)\left(\frac{E}{Z}\right)^{2}+P * Q}$

Donde:

n: Tamaño de muestra

N: Población de pacientes con cada uno de los distintos tipos de cáncer 
P: Proporción de población en riesgo para cada uno de los distintos tipos de cáncer (RMI)

Q: Complemento de $\mathrm{P}(\mathrm{Q}=1-\mathrm{P})$

E: Error estimado $\mathrm{E}=0.035(3.5 \%)$

Z: $95 \%$ de confianza (1.96)

Así mismo, se incrementó el tamaño muestral en un $10 \%$ ante la posibilidad de no encontrar la totalidad de los casos escogidos. A este nuevo tamaño de muestra se le denominó tamaño de muestra final. (Tabla 1)

\section{Procedimiento de selección de los casos de la muestra:}

La selección de los casos se realizó de manera sistemática y sólo en el caso de las neoplasias en las que fue necesario calcular tamaño de muestra. Para cada tipo de neoplasia se elaboró un marco muestral, conformado por todas las unidades de muestreo que forman nuestra población, posteriormente se ordenó estas unidades según hospital de procedencia y en base a este orden, se estableció la enumeración desde 1 hasta $\mathrm{N}$.

Se determinó nuestro intervalo de selección utilizando:

$\mathrm{K}=\frac{N}{n}$

Dónde: N: Tamaño de la población y n: Tamaño de la muestra

En todas las neoplasias se obtuvo un valor "K" no entero; lo que indica que estamos ante un muestreo sistemático circular; por lo tanto, se redondeó al número entero más próximo. Se seleccionó un número aleatorio "a" (arranque aleatorio) entre 1 y N sumándole la constante $\mathrm{K}$ para seleccionar los casos para la muestra de la siguiente manera:

$\mathrm{a} ; \mathrm{a}+\mathrm{k} ; \mathrm{a}+2 \mathrm{k} ; \mathrm{a}+3 \mathrm{k}$; 


\section{(4)}

\section{ACTA DE SUSTENTACIÓN}

En el día de hoy se reúne el jurado integrado por:

\begin{tabular}{|l|l|}
\hline Presidente & Alonso Soto Tarazona \\
\hline Jurado & Germán Alvarado Cutipa Flores \\
\hline Jurado & Edward Mezones Holguin \\
\hline
\end{tabular}

para evaluar la sustentación de:

$\mathbf{x}$ Tesis $\square$ Proyecto Profesiona

Expedientes

\section{titulado: RAZÓN MORTALIDAD-INCIDENCIA COMO INDICADOR DE SUPERVIVENCIA DE CÁNCER A CINCO AÑOS EN LIMA METROPOLITANA}

desarrollado por:

Alejandra Cordero Morales Karoline Andrea Stenning Persivale Maria Jose Savitzky Franco

asesorado por:

Eddy Segura Paucar Eduardo Payet Meza

para optar por el título

Médico Cirujano

profesional de:

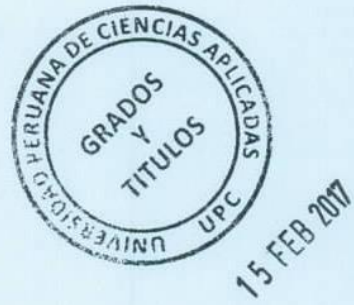

Después de haber escuchado la exposición, así como las respuestas a las preguntas formuladas en la defensa, el jurado concluye que el/los graduado(s) ha(n) demostrado estar preparado(s) para iniciar el ejercicio profesional. Por lo tanto, teniendo en cuenta los rangos de calificación siguiente:

/ Aprobado / Notable / Sobresaliente / Summa Cum Laude / Desaprobado /

el jurado otorga el siguiente resultado a:

\begin{tabular}{|l|c|}
\hline \multicolumn{1}{|c|}{ Estudiante } & Calificación \\
\hline Alejandra Cordero Morales & SP nOSDDO \\
\hline Karoline Andrea Stenning Persivale & SP NOBDDO \\
\hline Maria Jose Savitzky Franco & SP NOBDDO \\
\hline
\end{tabular}

Dado en la ciudad de Lima a los Ogdías del fnes de febrero de 2017.
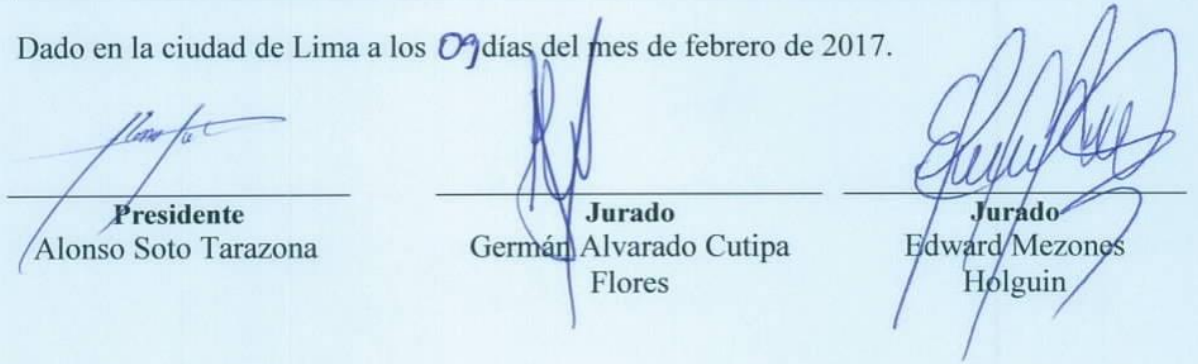
UPC

Ref. PI0202-2015: Relación Mortalidad-Incidencia como indicador de supervivencia de cáncer en Lima Metropolitana.

\section{Estimadas alumnas:}

En atención a la remisión del Protocolo indicado, tengo a bien hacer de su conocimiento que el Comité de Ética e Investigación (CEI) ha determinado aprobar el estudio tal como ha sido| replanteado.

Se les recuerda que el plazo de aprobación tiene una duración de 18 meses contados a partir de la fecha de esta carta, la que puede ser renovada luego de la presentación del informe anual de avances.

Sin otro particular, quedo de ustedes.

Atentamente.

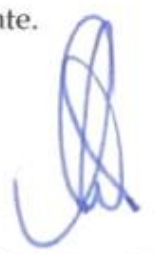

Dr. Aldo Vivar Mendoza

Presidente del Comité de Ética

Facultad de Ciencias de la Salud

d.Carta de aprobación de Comité de Ética 
e. Carta de cambio de título de la Tesis

\section{Modificación de título de tesis}

Sres. Grados y Títulos,

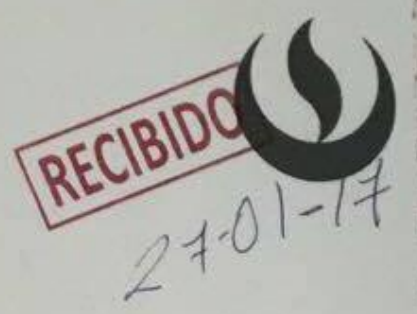

Mediante la presente se comunica la modificación del título de tesis de los alumnos: Alejandra Cordero Morales (U201010920), Karoline Stenning Persivale (U200912036) y Maria Jose Savitzky Franco (U200910978). El motivo del cambio es por recomendación de los asesores y para el mejor entendimiento del estudio.

Esta modificación no cambia el "tema" de tesis ni su contenido.

Título presente: "Relación Mortalidad-Incidencia como indicador de supervivencia de cáncer en Lima Metropolitana"

Nuevo Titulo: "Razón Mortalidad-Incidencia como indicador de supervivencia de cáncer a cinco años en Lima Metropolitana"

Atentamente,
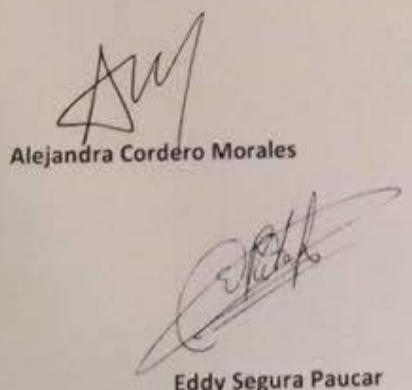

Asesor de tesis
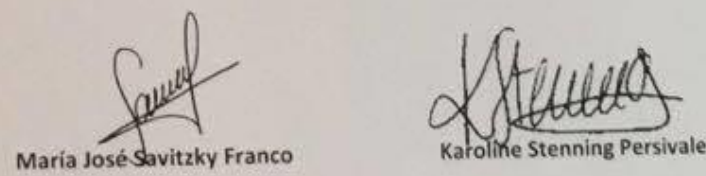

Dr. Mahuet Gutierrez Sierra

Director de Carrera, Escuela de Medicina 